\title{
LETTER
}

\section{Ribavirin is effective against drug-resistant H7N9 influenza virus infections}

\section{Dear Editor,}

In February and March 2013, a novel influenza A (H7N9) virus emerged in China, causing an acute respiratory distress syndrome and occasionally multiple organ failure with high fatality rates in humans (Li et al., 2014). A total of 681 laboratory-confirmed cases and 275 deaths have been reported as of November 13th, 2015, with a fatality rate of 40\% (http://www.who.int/influenza/human_animal_interface/ HAl_Risk_Assessment/en/). H7N9 has been evolving and established amongst chickens in China over the past two years with occasional human infections (Lam et al., 2015; Su et al., 2015), thus posing a threat to public health. In the absence of an annually-updated effective vaccine, antiviral drugs constitute the first line of defense against H7N9 infections. H7N9 viruses already possess natural resistance to M2-ion channel blockers (amantadine and rimantadine) when it first emerged in 2013 (Gao et al., 2013). Therefore neuraminidase inhibitors (NAls), which include oseltamivir (TamifluH), zanamivir (RelenzaH) and peramivir constitute the main antiviral drugs against $\mathrm{H} 7 \mathrm{~N} 9$ infections (Hu et al., 2013; Wu et al., 2013). However, treatment with NAls against H7N9 infections has resulted in the emergence of drug-resistant mutant viruses, as soon as 1 9 days after administration (Gao et al., 2013; Hu et al., 2013). Moreover, the first H7N9 isolate (A/Shanghai/1/2013(H7N9), SH-H7N9) was resistant to oseltamivir (Gao et al., 2013).

It is therefore necessary to investigate whether other classes of drugs can control H7N9 infections. A recent study shows that the RNA polymerase 2 (PB2) gene of the H7N9 virus is critical for virulence in mammals (Bi et al., 2015). Ribavirin is a well-characterized, broad-spectrum nucleoside inhibitor used to halt the synthesis and capping of viral RNA and mRNA, respectively, by the viral RNA-dependent RNA polymerase (Crotty et al., 2000). Ribavirin is approved for treating infections with Hepatitis C virus and respiratory syncytial virus (Graci and Cameron, 2006). Moreover, ribavirin is effective by itself or in combination with NAls and/or M2-ion channel blockers against H1N1, H3N2 and H5N1 influenza infections (Smee et al., 2005; llyushina et al., 2008; Nguyen et al., 2009). Here, we want to investigate whether ribavirin is effective by itself against H7N9 virus infections, especially to virus mutants that have developed resistance to NAls. To compare the efficacy of ribavirin to that of NAls, zanamivir was used as a positive control.

To determine the effectiveness of the ribavirin against $\mathrm{H} 7 \mathrm{~N} 9$ viruses in vitro, NAI-sensitive (A/Anhui/1/2013, AHH7N9) and -resistant (SH-H7N9) viruses were used in this study. The $50 \%$ effective virus-inhibitory concentration $\left(E C_{50}\right)$ value was used to evaluate the antiviral functions of ribavirin. Serial two-fold dilutions of ribavirin were used to test the $\mathrm{EC}_{50}$ against $\mathrm{AH}-\mathrm{H} 7 \mathrm{~N} 9$ and $\mathrm{SH}-\mathrm{H} 7 \mathrm{~N} 9$ on MadinDarby Canine Kidney (MDCK) epithelial cells, respectively. The results showed that ribavirin was effective against both $\mathrm{AH}-\mathrm{H} 7 \mathrm{~N} 9$ and $\mathrm{SH}-\mathrm{H} 7 \mathrm{~N} 9$ with an $\mathrm{EC}_{50}$ of $0.01 \sim 0.02 \mathrm{mg} / \mathrm{mL}$ (3 4 nmol) (Table 1), implying that ribavirin could be utilized against both NAl-sensitive and -resistant H7N9 viruses. In addition, the ribavirin at a dose of $\sim 300$ folds of the calculated $\mathrm{EC}_{50}$ is also safe in vitro without significant cytotoxicity.

In vitro studies showed that the ribavirin is effective to both the NAl-sensitive (AH-H7N9) and -resistant (SH-H7N9) viruses. To further study antiviral function in vivo, the efficacy of ribavirin against H7N9 infection was then tested in a mouse animal model. To confirm the antiviral functions of ribavirin, zanamivir was selected as a positive drug control.

The survival, percentage weight change and clinical symptoms in the animals after challenge were monitored over the course of the experiment (14 days). All animals infected with $\mathrm{AH}-$ H7N9 displayed ruffled fur, loss of activity and body weight loss beginning 2 days post-infection (d.p.i.). Mice treated with placebo did not survive and had weight loss of over $35 \%$ (Fig. 1A and $1 \mathrm{~B}$ ). Mice treated with ribavirin or zanamivir also experienced weight loss of up to around $30 \%$, but all mice in each group gradually recovered and survived (Fig. $1 \mathrm{~A}$ and $1 \mathrm{~B}$ ). In general, the clinical signs of mice in the ribavirin and zanamivir groups were milder than that of the placebo group. No significant changes were observed from mice in the mock-infection group.

Five mice from each group were euthanized at 3 d.p.i. and the lung virus titers (LVTs) were test in MDCK cells, with the results presented as $50 \%$ tissue culture infective dose $\left(T C I D_{50}\right)$. The results showed that the LVTs from mice treated with either the ribavirin or zanamivir were 10-fold lower than that of the sterile phosphate-buffered saline (PBS) placebo group $(P<0.5)$ (Fig. 1C). Interestingly, ribavirin- 
Table 1. $E C_{50}$ values of ribavirin against $\mathrm{H} 7 \mathrm{~N} 9$ virus

\begin{tabular}{lll}
\hline Viruses & \multicolumn{2}{l}{ Ribavirin $( \pm S D)$} \\
\cline { 2 - 3 } & $\mathrm{mg} / \mathrm{mL}$ & Effective dose $(\mathrm{nmol})$ \\
\hline AH-H7N9 & $0.0166( \pm 0.0029)$ & $3.3964( \pm 0.5864)$ \\
SH-H7N9 & $0.0182( \pm 0.0021)$ & $3.7186( \pm 0.4288)$ \\
\hline
\end{tabular}

treated mice displayed lower LVTs than that of zanamivirtreated animals, but the values were not statistically significant $(P>0.5)$ (Fig. 1C).

In conclusion, ribavirin displayed antiviral activities comparable to zanamivir against H7N9 virus infections in vitro and in vivo, and the data support the use of ribavirin against NAI-resistant $H 7 N 9$ virus infections. Since it had been shown in previous studies that $\mathrm{SH}-\mathrm{H} 7 \mathrm{~N} 9$ containing the NAI-

A

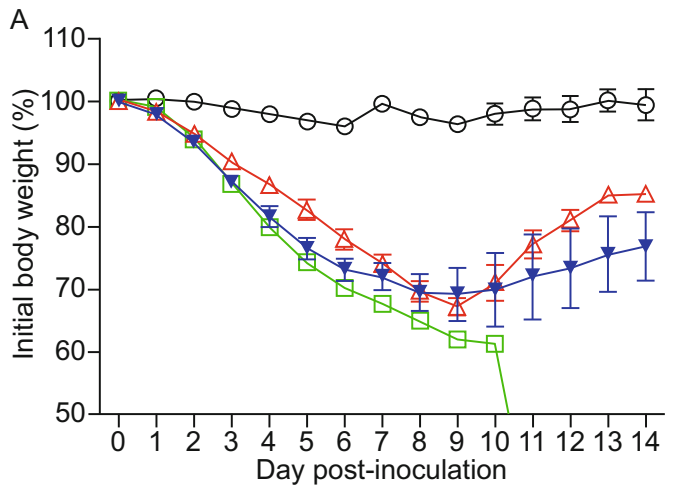

B
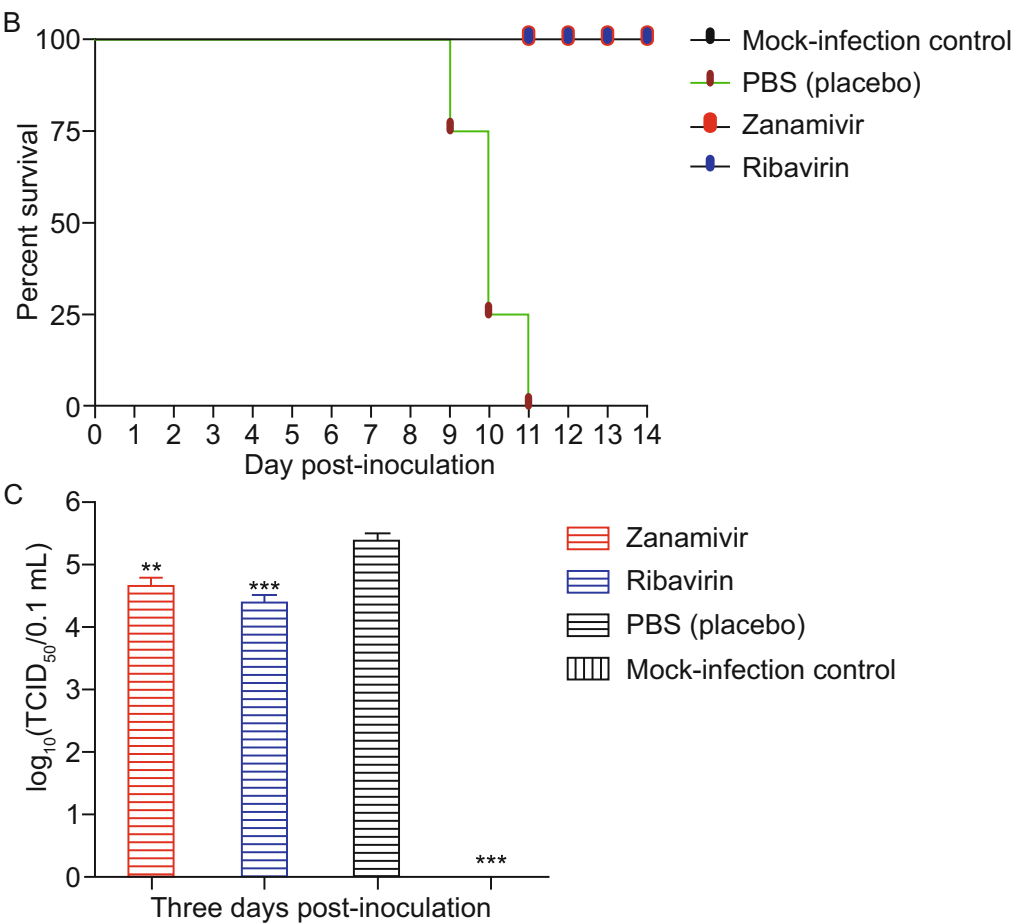

- Mock-infection control

$\square$ PBS (placebo)

$\triangle$ Zanamivir

$\rightarrow$ Ribavirin

Figure 1. Efficacy of ribavirin in mice infected with AH-H7N9. Mice $(n=9)$ were given ribavirin, zanamivir or PBS (placebo) twice daily, respectively. Animals were inoculated i.n. with $10 \times \mathrm{LD}_{50}$ of $\mathrm{AH}-\mathrm{H} 7 \mathrm{~N} 9$ in $50 \mu \mathrm{L}$ PBS at one day after the initiation of treatment, and an untreated group was mock-challenged with an equal volume of PBS as control. Survival and body weights were monitored daily over a 14-day observation period and expressed as percentages of the initial values (A). The mortality rate was calculated from the survival curve of each group (B). Five mice from each group were euthanized at 3 d.p.i. LVTs were quantified in MDCK cells, and expressed as $\log _{10} \mathrm{TCID}_{50} / 0.1 \mathrm{~mL}(\mathrm{C})$. The data are presented as the mean $\pm \mathrm{SD}$. Statistical analysis on the LVTs were performed with a paired-sample $t$-test $\left({ }^{*}, P<0.05 ;{ }^{* *}, P<0.01 ;{ }^{* * *}, P<0.001\right)$. 
resistant K224 mutation ( $\mathrm{H} 3$ numbering) on the neuraminidase (NA) gene reverts back to the NAI-sensitive R224 mutation in vivo (Yen et al., 2014), as well as the relatively lower replication ability in vitro compared to $\mathrm{AH}-\mathrm{H} 7 \mathrm{~N} 9$ (the NAl-sensitive virus) (Wu et al., 2013), only AH-H7N9 was used for the studies in mice.

Notably, administration of ribavirin via the intranasal (i.n.) route was still efficacious against severe influenza virus infections without any of the negative side effects associated with oral or intravenous administration (Gilbert and McLeay, 2008). The present study showed that the i.n. route is also effective in the case of ribavirin against $\mathrm{H} 7 \mathrm{~N}$ 9 virus infection. Human infections with H7N9 mainly originate from contact with infected poultry or contaminated materials in live poultry markets (Gao, 2014), and administration of ribavirin into the nasal mucosa would be an effective strategy to mitigate the risk of H7N9 infections in humans.

\section{FOOTNOTES}

This work was supported by the National Basic Research Program (973 Program) (Nos. 2013CB531502 and 2014CB542503) and the National Natural Science Foundation of China (Grant No. 31402196). Yi Shi is supported by the Excellent Young Scientist Program of the Chinese Academy of Sciences and the Youth Innovation Promotion Association CAS (2015078). Gary Wong is the recipient of a Banting Postdoctoral Fellowship from the Canadian Institutes of Health Research (CIHR) and the President's International Fellowship Initiative from the Chinese Academy of Sciences (CAS).

Ribavirin (National Drug Approval No. H20043189) was obtained from Penglai Nuokang Pharmaceutical Co., Ltd. (Penglai city, Shandong Province, China). Zanamivir carboxylate was purchased from Shandong Xiya Chemical Industry Co., Ltd. (Shandong Province, China).

Yuhai Bi, Gary Wong, Yingxia Liu, Lei Liu, George F Gao and Yi Shi declare that they have no conflict of interest. All institutional and national guidelines for the care and use of laboratory animals were followed.

Yuhai $\mathrm{Bi}^{1,2,3 凶}$, Gary Wong ${ }^{1}$, Yingxia Liu ${ }^{2}$, Lei Liu ${ }^{2}$, George F. Gao ${ }^{1,2,3,4,5}$, Yi Shi ${ }^{1,2,3,4 \bowtie}$

${ }^{1}$ CAS Key Laboratory of Pathogenic Microbiology and Immunology, Institute of Microbiology, Chinese Academy of Sciences, Beijing 100101, China

2 Shenzhen Key Laboratory of Pathogen and Immunity, State Key Discipline of Infectious Disease, Shenzhen Third People's Hospital, Shenzhen 518112, China

${ }^{3}$ Center for Influenza Research and Early-warning (CASCIRE), Chinese Academy of Sciences, Beijing 100101, China

${ }^{4}$ Research Network of Immunity and Health (RNIH), Beijing Institutes of Life Science, Chinese Academy of Sciences, Beijing 100101, China

${ }^{5}$ Collaborative Innovation Center for Diagnosis and Treatment of Infectious Disease, Zhejiang University, Hangzhou 310003, China $\triangle$ Correspondence: beeyh@im.ac.cn (Y. Bi), shiyi@im.ac.cn (Y. Shi)

\section{OPEN ACCESS}

This article is distributed under the terms of the Creative Commons Attribution 4.0 International License (http://creativecommons.org/ licenses/by/4.0/), which permits unrestricted use, distribution, and reproduction in any medium, provided you give appropriate credit to the original author(s) and the source, provide a link to the Creative Commons license, and indicate if changes were made.

\section{REFERENCES}

Bi Y, Xie Q, Zhang S, Li Y, Xiao H, Jin T, Zheng W, Li J, Jia X, Sun L et al (2015) Assessment of the internal genes of influenza $A$ (H7N9) virus contributing to high pathogenicity in mice. J Virol 89:2-13

Crotty S, Maag D, Arnold JJ, Zhong WD, Lau JYN, Hong Z, Andino R, Cameron CE (2000) The broad-spectrum antiviral ribonucleoside ribavirin is an RNA virus mutagen. Nat Med 6:1375-1379

Gao GF (2014) Influenza and the live poultry trade. Science 344:235

Gao R, Cao B, Hu Y, Feng Z, Wang D, Hu W, Chen J, Jie Z, Qiu H, $\mathrm{Xu} \mathrm{K}$ et al (2013) Human infection with a novel avian-origin influenza A (H7N9) virus. N Engl J Med 368:1888-1897

Gilbert BE, McLeay MT (2008) MegaRibavirin aerosol for the treatment of influenza A virus infections in mice. Antiviral Res 78:223-229

Graci JD, Cameron CE (2006) Mechanisms of action of ribavirin against distinct viruses. Rev Med Virol 16:37-48

Hu YW, Lu SH, Song ZG, Wang W, Hao P, Li JH, Zhang XN, Yen HL, Shi BS, Li T et al (2013) Association between adverse clinical outcome in human disease caused by novel influenza A H7N9 virus and sustained viral shedding and emergence of antiviral resistance. Lancet 381:2273-2279

Ilyushina NA, Hay A, Yilmaz N, Boon AC, Webster RG, Govorkova EA (2008) Oseltamivir-ribavirin combination therapy for highly pathogenic H5N1 influenza virus infection in mice. Antimicrob Agents Chemother 52:3889-3897

Lam TTY, Zhou BP, Wang J, Chai YJ, Shen YY, Chen XC, Ma C, Hong WS, Chen $Y$, Zhang $Y J$ et al (2015) Dissemination, divergence and establishment of H7N9 influenza viruses in China. Nature 522:102-U265

Li Q, Zhou L, Zhou M, Chen Z, Li F, Wu H, Xiang N, Chen E, Tang F, Wang $D$ et al (2014) Epidemiology of human infections with avian influenza $\mathrm{A}(\mathrm{H} 7 \mathrm{N9})$ virus in China. N Engl J Med 370:520-532

Nguyen JT, Hoopes JD, Smee DF, Prichard MN, Driebe EM, Engelthaler DM, Le MH, Keim PS, Spence RP, Went GT (2009) Triple combination of oseltamivir, amantadine, and ribavirin displays synergistic activity against multiple influenza virus strains in vitro. Antimicrob Agents Chemother 53:4115-4126

Smee DF, Bailey KW, Wong MH, Sidwell RW (2005) Activities of oseltamivir and ribavirin used alone or in combination against infections caused by mouse-adapted recent isolates of influenza $A$ and $B$ viruses. Antiviral Res 65:A61-A61

Su S, Bi Y, Wong G, Gray GC, Gao GF, Li S (2015) Epidemiology, evolution, and recent outbreaks of avian influenza virus in china. J Virol 89:8671-8676

Wu Y, Bi Y, Vavricka CJ, Sun X, Zhang Y, Gao F, Zhao M, Xiao H, Qin C, He J et al (2013) Characterization of two distinct 
neuraminidases from avian-origin human-infecting H7N9 influenza viruses. Cell Res 23:1347-1355

Yen HL, Zhou J, Choy KT, Sia SF, Teng O, Ng IH, Fang VJ, Hu Y, Wang W, Cowling BJ et al (2014) The R292K mutation that confers resistance to neuraminidase inhibitors leads to competitive fitness loss of A/Shanghai/1/2013 (H7N9) influenza virus in ferrets. J Infect Dis 210:1900-1908

Yuhai $\mathrm{Bi}$ and Gary Wong contributed equally to this study.

Electronic supplementary material The online version of this article (doi:10.1007/s13238-016-0287-0) contains supplementary material, which is available to authorized users. 